\title{
Sub-kHz linewidth VECSEL for cold atom experiments
}

\author{
P.H. Moriya, J.E. Hastie \\ Institute of Photonics, Department of Physics, SUPA, University of Strathclyde, \\ The Technology and Innovation Centre, 99 George Street, Glasgow G1 1RD, UK \\ Author e-mail address: paulo.moriya@strath.ac.uk
}

\begin{abstract}
We report sub-kHz linewidth operation of a frequency-stabilized, AlGaInP-based vertical-external-cavity surface-emitting laser (VECSEL) at $689 \mathrm{~nm}$, suitable for Strontium cold atom experiments. $170 \mathrm{~mW}$ was emitted with linewidth $\leq 200 \mathrm{~Hz}$, determined via an optical beat note measurement.

OCIS codes: (140.3460) Lasers; (250.5960) Semiconductor lasers; (250.7270) Vertical emitting lasers; (140.3320) Laser cooling; (140.3425) Laser stabilization; (140.3570) Lasers, single-mode; (140.7300) Visible lasers; (140.7010) Laser trapping.
\end{abstract}

\section{Introduction}

Stable, high power, narrow linewidth, and tunable laser sources are crucial for a wide range of applications in several scientific and industrial fields. In particular they are required for the development of quantum technologies based on cold atoms with transitions at optical frequencies [1]. In these experiments many of the current systems are based on diode lasers that require stabilization to sophisticated and expensive reference cavities, with ultra-high stability and finesse, to reduce the free-running linewidth from hundreds of kHz. Further, depending on the required emission wavelength, these systems often do not provide sufficient optical power such that amplifiers or injection lock schemes need to be implemented, particularly if nonlinear frequency conversion is also to be implemented.

In this context VECSELs, also known as semiconductor disk lasers (SDLs), have demonstrated great potential due to their spectral flexibility (from UV to IR), high beam quality and brightness, and suitability for low noise single frequency operation [2,3]. Potential for miniaturization is another attractive feature as these lasers are compact, with any frequency conversion and filtering performed intracavity. Linewidths of a few $\mathrm{kHz}$ have been demonstrated over timescales of the order of $1 \mathrm{~s}[2,4,5]$, but sub-kHz linewidths, such as those required for narrow atom cooling and clock transitions, have yet to be reported. Here we report frequency stabilisation of an AlGaInPbased VECSEL locked to a standard commercial air-spaced Fabry-Perot cavity, achieving sub-kHz linewidth with powers up to $170 \mathrm{~mW}$. The emission wavelength can be tuned from 685 to $693 \mathrm{~nm}$, suitable for use as part of Strontium cold atom experiments; specifically for targeting the second-stage cooling transition used for Strontium magneto-optical traps: $689.4488968 \mathrm{~nm}(7.6 \mathrm{kHz})[5,7]$.

\section{The VECSEL gain structure and laser operation}

The VECSEL active region is based on compressively-strained GaInP quantum wells, grouped in pairs, separated by AlGaInP barriers grown on the top of an AlGaAs distributed Bragg reflector (DBR) [5]. The gain structure is designed to be pumped at $532 \mathrm{~nm}$ with direct emission centered at $689 \mathrm{~nm}$. Laser oscillation is achieved in a 2mirror (linear), cavity (see inset, Figure 1a) composed of the DBR and a $2 \%$ plano-concave output coupler $($ ROC $=$ $100 \mathrm{~mm}$ ), mounted on a piezoelectric transducer (PZT) for active stabilization. Inside the cavity, a Brewster-angled quartz plate is used as a birefringent filter (BRF) for wavelength tunability. Single frequency operation with output powers up to $170 \mathrm{~mW}$ at $689(4) \mathrm{nm}$ with free-running linewidth of $300 \mathrm{kHz}$ are reached when pumped with $3 \mathrm{~W}$ at $532 \mathrm{~nm}$ from a commercial VECSEL (Coherent Verdi G).

Frequency stabilization is performed via the Pound-Drever-Hall (PDH) technique [6], where part of the laser beam is frequency modulated by an electro-optic modulator (EOM; FM $=74 \mathrm{MHz}$ ) and coupled to a commercial Fabry-Perot cavity (air-spaced, confocal, finesse $=1000$ and free spectral range $=300 \mathrm{MHz}$ ) used as a reference. The reflected light is captured by a fast photodetector to create a correction signal which is sent back to the PZT in the laser cavity, closing the feedback loop. The linewidth is thus reduced to a sub-kHz regime while maintaining the same output power.

\section{Frequency noise characterization}

The frequency noise and the linewidth of the laser while actively stabilized to the commercial Fabry-Perot cavity are analyzed here using two methods. The first method consists in calculating a power spectral density (PSD) of the residual error signal of the feedback loop recorded over a set sampling time. This technique is widely used to estimate laser linewidth relative to a suitable reference (see e.g. $[4,5,8]$ ), particularly when no other laser is available, or where a self-heterodyne measurement is impractical [5,9], and has been the preferred method of characterizing stabilized VECSELs to date. Figure 1a shows the frequency noise PSD obtained for a $2 \mathrm{~s}$ sampling 
time from which we deduced the shape of the optical field and extracted a relative linewidth of $65 \mathrm{~Hz}$. Some peaks are located at frequencies higher than $10 \mathrm{kHz}$ and are due to electronic oscillations, which were kept as low as possible by limiting the gain of the servo electronics. The majority of the noise is compressed in the $0.1-1 \mathrm{kHz}$ region which indicates the presence of pure and thermally-induced mechanical noises in our experimental setup. The second method used was an optical beat note measurement with another narrow linewidth laser: a commercial external cavity diode laser (ECDL) with tapered amplifier (Toptica TA Pro) with output power of $160 \mathrm{~mW}$ locked via PDH to a temperature-stabilized and high finesse ultra-stable vertical reference cavity (finesse $=300000$ and free spectral range $=1 \mathrm{GHz}$ ), with both mirror substrates and spacers made of ultralow expansion (ULE) glass kept under vacuum, with expected linewidth of $10 \mathrm{~Hz}$. The VECSEL and ECDL system were located in adjacent laboratories, with the latter coupled via optical fiber to deliver the signal for the beat note measurement. The overlapped beams were captured by a fast photodetector and analyzed with an electronic spectrum analyzer. Figure 1(b) shows the beat note peak at $102 \mathrm{MHz}$ with a full-width at half-maximum of $200 \mathrm{~Hz}$. It is important to note that this measurement provides an upper limit for the linewidth and includes any uncorrelated mechanical, electronic and thermal fluctuations that will broaden the beat note between the two separate laser systems.
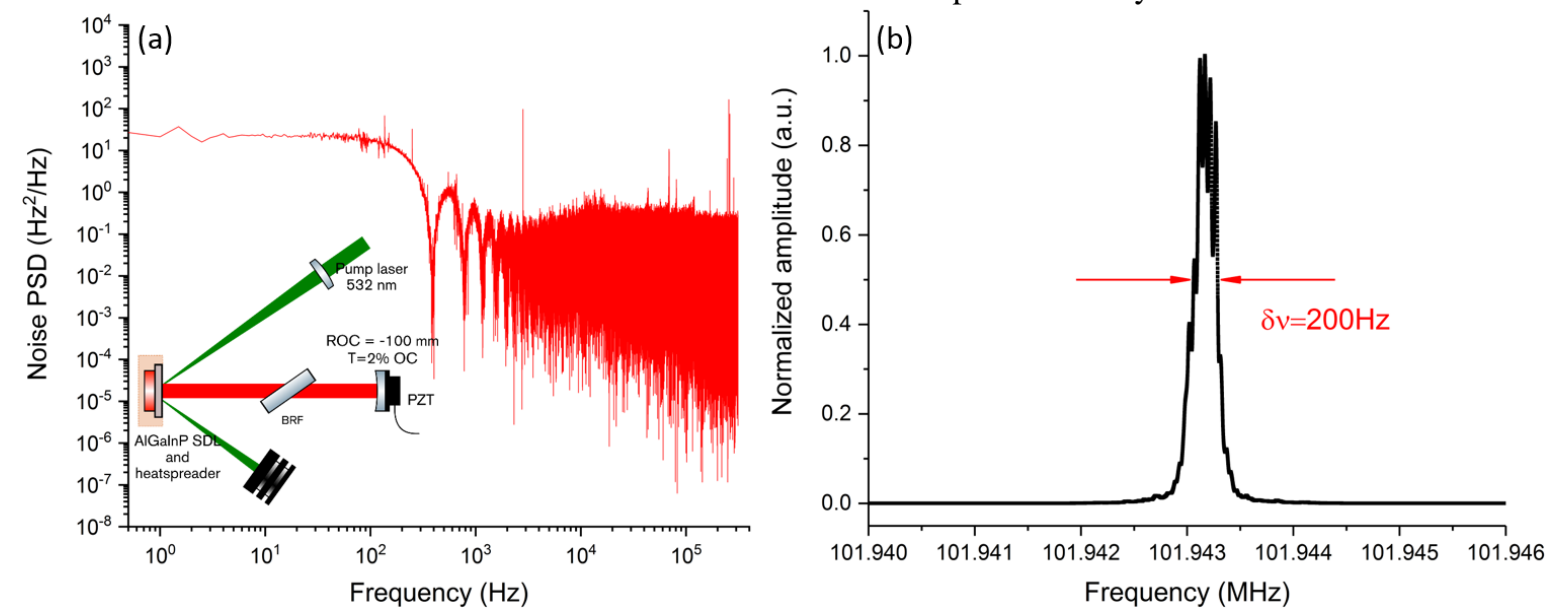

Figure 1 - Frequency noise analysis of the AlGaInP-based VECSEL system when locked to a reference cavity via the PDH technique. (a) Calculated PSD of the residual error signal of the feedback loop recorded over a sampling time of $2 \mathrm{~s}$. From this a relative linewidth of $65 \mathrm{~Hz}$ is estimated. Inset: Schematic of the AlGaInP-based VECSEL. (b) Optical beat note measurement against a commercial laser system locked to an ultra-stable, high finesse reference cavity (finesse $=300000$, free spectral range $=1.5 \mathrm{GHz}$ ). The optical beat note signal is seen centered around $101.943 \mathrm{MHz}$ and has a FWHM of $200 \mathrm{~Hz}$.

\section{Conclusions and next steps}

We have demonstrated a $\leq 200 \mathrm{~Hz}$ linewidth, single-frequency VECSEL locked to a commercial air-spaced FabryPerot reference cavity. To our knowledge this is the narrowest linewidth reported from a VECSEL, but still at least two orders of magnitude larger than the Schawlow-Townes-Henry limit [10,11]. In future work towards a more compact and portable VECSEL system for cold atom optics we intend to optimize the performance of the VECSEL system when subject to a noisier optical pump, such as blue or even green laser diodes.

\section{Acknowledgement}

We thank the group of Kai Bongs at the Midlands Ultracold Atoms Research Centre, University of Birmingham, UK for the use of the locked diode laser system used for the beat note measurement.

\section{References}

[1] L. Fallani \& A. Kastberg, "Cold atoms: A field enabled by light”, EPL 110, 53001 (2015).

[2] A. Garnache et al., "Design and properties of high-power highly coherent single-frequency VECSEL emitting in the near-to mid-IR for photonic applications," in Proceedings of SPIE Photonics West 7919, 791914(11) (2011).

[3] M. Guina et al., "Optically pumped VECSELs: review of technology and progress", Journal of Physics D 50, 383001 (2017).

[4] M.A. Holm et al., “Actively stabilized single-frequency vertical-external-cavity AlGaAs laser”, IEEE Phot. Tech. Lett. 11, 1551 (1999).

[5] D. Paboeuf \& J. E. Hastie, "Tunable narrow linewidth AlGaInP semiconductor disk laser for Sr atom cooling applications", Appl. Opt. 55, 19, 4980 (2016).

[6] E.D. Black, “An introduction to Pound-Drever-Hall laser frequency stabilization”, American Journal of Physics 69, 79 (2001).

[7] T. Ido et al., "Precision Spectroscopy and Density-Dependent Frequency Shifts in Ultracold Sr", Phys. Rev. Lett. 94, 153001(4) (2005).

[8] G. Di Domenico et al., "Simple approach to the relation between laser frequency noise and laser line shape", Appl. Opt. 49, 4801 (2010).

[9] A. Rantamäki et al.,"4.6-W Single Frequency Semiconductor Disk Laser With <75-kHz Linewidth”, IEEE Phot. Tech. Lett. 24, 1378 (2012).

[10] A.L. Schawlow \& C.H. Townes, "Infrared and optical masers", Phys. Rev. 112, 1940-1949 (1958).

[11] C. Henry, "Theory of the linewidth of semiconductor lasers", IEEE J. Quantum Electron. 18, 259-264 (1982). 